\title{
Solving High Dimensional and Complex Non-convex Programming Based on Improved Quantum Artificial Fish Algorithm
}

\author{
Yang $\mathrm{Hu}^{1}$, Tingsong $\mathrm{Du}^{1,2, *}$, Jinhui $\mathrm{Wu}^{1}$, Xianhui $\mathrm{Liu}^{1}$, Deyi $\mathrm{Li}^{2}$ and Wenwu $\mathrm{LI}^{3}$ \\ ${ }^{I}$ Institute of Nonlinear and Complex Systems, China Three Gorges University, Yichang 443002, China \\ ${ }^{2}$ Hubei Province Key Laboratory of System Science in Metallurgical Process, Wuhan 430065, China \\ ${ }^{3}$ College of Electrical Engineering and Reusable Energy, China Three Gorges University, Yichang 443002, China
}

\begin{abstract}
An improved quantum artificial fish swarm algorithm is proposed in this paper. Based on that quantum computing have exponential acceleration for heuristic algorithm, by examining eight most recent patents and some literatures in the area of artificial fish swarm algorithm and quantum computing. The new algorithm uses qubits to code artificial fish and quantum revolving gate, preying behavior, following behavior and variation of quantum artificial fish to update the artificial fish for searching for optimal value. Then, apply the new algorithm, the basic artificial fish swarm algorithm and the global edition artificial fish swarm algorithm to the simulation experiment high dimensional and complex non-convex programming respectively. The simulation results show that the improved algorithm can escape from the local extremum effectively, and has higher convergence speed and precision.
\end{abstract}

Keywords: Artificial fish swarm algorithm, non-convex programming, quantum computing.

\section{INTRODUCTION}

With the continuous development and progress of science and technology, a growing number of large-scale complex engineering optimization problems using traditional optimization methods is difficult to obtain the globally optimal value. Modern intelligent optimization algorithm is convenient to deal with constraints and shows global search ability. However, every intelligent optimization algorithm which has the ability to solve functional optimization problems contains advantages and disadvantages. How to effectively combine the merits of different intelligent algorithms for improving the overall performance is a hot topic for researchers [1-3]. Patent CN 102,708,248, entitled "Dispatching function optimization method based on multi-objective genetic algorithm" [4] invented a method based on a multiobjective genetic algorithm that comprises of setting the form of a dispatching function; setting decision variables; and calculating dispatching rules by multi-objective particle swarm optimization (MOPSO).

Basic Artificial Fish Swarm Algorithm (BAFSA) is a swarm intelligence optimization algorithm based on animal behavior [5]. It mainly simulates foraging, huddling, following and random behavior, starting from the structure of the underlying behavior of a single fish and achieving the purpose of the group global optimization. The BAFSA is easy to implement and has good ability to obtain the global extremum and avoid falling into local minima. It is not sensitive to initial value and parameter selection, and has strong robustness and good convergence performance. But there are also some influence search quality and efficiency of weakness: the poor ability to keep balance of exploration and development, large blindness of the algorithm running late search, slow arithmetic speed and low accuracy of optimization results.

Quantum computing is different from the traditional calculation model of classical physics, which has incomparable advantages such as quantum super parallelism, exponential storage capacity, etc [6]. In the Patent US 5,940,193 entitled "General purpose quantum computing"[7], the method and apparatus are provided for a general purpose photonic computer. A data signal is input through an encoder to encode such signal with an instruction. The encoded signal is transmitted by means of a laser beam to an input buffer where it interferes with a reference beam so as to form an interference pattern therein as a hologram, IPH. A read beam is directed through the IPH and through a decoder which reads the instruction as having an OP Code, data source and destination. The decoded instruction is forwarded on the read beam to ALU spin media which respond to the instruction by flipping spins between two energy levels, in one or more sequence of data patterns which are read or measured by one or more sensors. Such sensors can be RF, microwave or optical sensors, which sensors output Radix $=2$ or digital data signals for, e.g. storage, display or further processing as desired. Thus the present invention describes a novel exploitation of photon-induced quantum-mechanical spin transitions in spin media. The input signal can be from a keyboard, camera, bar code or other input source. Its essence is the use 
of superposition of quantum state. The combination of quantum computing and intelligent optimization algorithm injected new life into the intelligent optimization computing source, through using quantum computing in new mode of information representation and information processing [8, 9]. Patent US 7,447,719 entitled "Quantum computing method and quantum computer" [10] describes $(\mathrm{N}+1)$ number of physical systems each having five energy levels $|0>| 1>,, \mid 2>$, $\mid 3>$, and $\mid 4>$, a qubit being expressed by $\mid 0>$ and $\mid 1>$, are provided in an optical cavity having a cavity mode resonant with $|2>-| 3>$, such that an $\mathrm{N}$ number of control systems and a target system are prepared. The target system is irradiated with light pulses resonant with $|0>-| 4>,|1>-| 4>$, and $|2>-| 4>$ to change a superposed state $\mid c>$ to $\mid 2>$. All of the physical systems are irradiated with light pulses resonant with $|0>-| 3>$ and $|1>-| 3>$, and a phase of the light pulse resonant with the target system is shifted by a specific value dependent on a unitary transformation $U$. The target system is irradiated with light pulses resonant with $|0>-| 4>,|1>-| 4>$, and $|2>-| 4>$, with a phase difference between them being set to a specific value dependent on the unitary transformation $U$, to return $\mid 2>$ to $|c\rangle$.And it can study the design method of intelligent optimization algorithm from another angle of view to enrich the theory of intelligent computing and improve the traditional method of intelligent searching performance as a whole.

In this paper, the improved quantum artificial fish swarm algorithm (IQAFSA) is proposed in view of the slow speed and low accuracy of BAFSA and randomness and blindness of quantum computing $[11,12]$ whose improvements are the coding way of the quits artificial fish, using quantum revolving door, artificial fish following, artificial fish preying and variation of update strategy to implement self-renewal, resulting in a new artificial fish. Finally, IQAFSA is applied to high dimensional and complex non-convex programming. Patent US 4,935,877 entitled "Non-linear genetic algorithms for solving problems" [13] shows that the present invention is a non-linear genetic algorithm for problem solving, which makes use of intelligent algorithm to solve the non-convex problem. By simulation experiment with BAFSA and the global edition artificial fish swarm algorithm (GAFSA) [14]. Patent CN 101,515,338, entitled "Artificial fish-swarm algorithm based on overall information" [15] provides an artificial fish-swarm algorithm based on overall information, comprising of the followings steps: 1) firstly initializing settings, 2) calculating the fitness value of each artificial fish and recording the status of overall optimized artificial fish, 3) evaluating each artificial fish and selecting the behaviors to be acted by fish, including feeding, bunching, tailing, biting and jumping, 4) acting the behaviors selected by the artificial fish and updating the position information of the fish based on the overall and local information, 5) updating the status of overall optimized artificial fish, and 6) outputting the result if the condition of loop termination is met, or returning to the step 2). The invention improves the basic artificial fish-swarm algorithm and provides a new fish-swarm optimizing mode and biting and jumping behaviors of the artificial fish, reduces the complexity the algorithm enhances the overall optimizing capability of algorithm and increases the speed and precision of convergence of the algorithm. The simulation results show that IQAFSA has great superiority in function optimization, which has advantages of small fish scale, fast convergence speed and high precision optimization results.

\section{DESCRIPTION OF BAFSA}

Artificial fish implement self-renewal and obtain the optimal value mainly through the four behaviors, namely random, preying, swarming and following in the process of iterative calculation.

\section{i. AF-Random}

Random behavior is to select a state in the field of vision, and then move to the direction. It is actually a default behavior.

\section{ii. AF-prey}

Preying is a kind of the basic behavior of artificial fish which is an activity that tends to the direction of the high concentration of food.

$$
\left\{\begin{array}{l}
X_{\text {inext }}=x_{i k}+\operatorname{Random}(\text { step }) \frac{x_{j k}-x_{i k}}{\left\|X_{j}-X_{i}\right\|}, Y\left(X_{j}\right) \text { is better then } Y\left(X_{i}\right) \\
X_{\text {inext }}=x_{i k}+\operatorname{Random}(\text { step }), \text { else }
\end{array}\right.
$$

where $x_{j k}$ is $k$ element of state vector $X_{j} ; x_{i k}$ is $k$ elements of $X_{i} ; x_{\text {inext }}$ is the next step state vector; Random (step) is a random number between 0 and step. $k=1,2, \ldots$

\section{iii. AF-swarm}

AF-swarm refers that every fish moves as far as possible to the center of the adjacent partners in the process of swimming, and avoids overcrowding. Supposed that $X_{i}$ is the current state of artificial fish $i . n_{f}$ is the number of partners and $X_{c}$ is the center position in the current field $\left(d_{\mathrm{ij}}<\right.$ Visual ). If $\frac{Y_{c}}{n_{f}}>\delta Y_{i}$, it shows that there are more food around the partner and it's not too crowded. Then, the fish moves a step forward to the center position of this partner,

$$
X_{i}^{t+1}=X_{i}^{t}+\frac{X_{c}-X_{i}^{t}}{\left\|X_{c}-X_{i}^{t}\right\|} \cdot \text { Step } \cdot \text { Rand }() \text {. }
$$

Otherwise, the fish performs preying behavior. 


\section{iv. AF-follow}

AF-follow refers that the artificial fish moves to the visual range of the optimal direction. Supposed that $X_{i}$ is the current state of artificial fish $i . Y_{j}$ is the greatest partner in the current field $\left(d_{\mathrm{ij}}<\right.$ Visual $)$. If $\frac{Y_{j}}{n_{f}}>\delta Y_{i}$, it shows that there are more food around $X_{j}$ and it's not too crowded. Then the fish moves a step forward to the direction of $X_{j}$.

$X_{i}^{t+1}=X_{i}^{t}+\frac{X_{j}-X_{i}^{t}}{\left\|X_{j}-X_{i}^{t}\right\|} \cdot \operatorname{Step} \cdot \operatorname{Rand}()$.

Otherwise, the fish performs preying behavior.

In the function optimization, the assumption is that in a $D$-dimensional search space goal, there is $n$ artificial fish. $\mathrm{X}=\left(x_{1}, x_{2}, \ldots, x_{n}\right)$ is the state of artificial individual fish, where $x_{i}(i=1,2, \ldots, n)$ is the optimal control variable. $Y=f(X)$ is the food concentration of artificial fish at its current location, where $Y$ is the objective function value. $d_{i j}=\left\|X_{i}-X_{j}\right\|$ is the distance of the fish $i$ and the fish $j$. The idea of BAFSA is that it firstly initializes a swarm of artificial fish (random solutions), and then the fish implement self-renewal by four basic behaviors. Each of the artificial fish explores the current environment conditions (including the change of the objective function and partners). Then, selecting an appropriate behavior and making the optimal direction move fastest. Finally, artificial fish gather around several local minima, especially in some better extremum areas, which are generally able to rally more artificial fish, namely the optimal value of objective function.

\section{QUANTUM COMPUTING}

In quantum computing, Patent US 20,060,224,547 titled "Efficient simulation system of quantum algorithm gates on classical computer based on fast algorithm" [16], an efficient simulation system of quantum algorithm gates for classical computers with a Von Neumann architecture is described. In one embodiment, a Quantum Algorithm is solved using an algorithmic-based approach, wherein matrix elements of the quantum gate are calculated on de-mand. In one eobmdiment, a problem-oriented approach to implementing Grover's algorithm is provided with a termination condition determined by observation of Shan-non minimum entropy. In one embodiment, a Quantum Control.

Algorithm is solved by using a reduced number of quantum operations, physical media acting as the information storage unit is a two-state quantum system, referred to as quantum bit. Aquantum bit can be itself in a super position of two quantum states, represented by:

$$
|\phi\rangle=\alpha|0\rangle+\beta|1\rangle \text {, where } \alpha \text { and } \beta \text { are quantum bit prob- }
$$

ability amplitude. The probability of $|0\rangle$ is represented by $|\alpha|^{2}$, and the probability of $|1\rangle$ is represented by $|\beta|^{2}$, $|\alpha|^{2}+|\beta|^{2}=1$. Each quantum state in system can be expressed as the superposition of the basic state, as $|\alpha|^{2}$ and $|\beta|^{2}$ tend to be 1 or 0 , the quantum bit will converge to a state.

The update of the quantum bit needs to make use of the transformation of quantum gates, commonly including the xor gate, the controlled xor gate, Hadamard transform gate and the revolving gate. In this paper, we adopt the quantum revolving gate $[17,18]$, change its phase by interference and then change the basic state probability amplitude, namely

$$
U(\theta)=\left[\begin{array}{rr}
\cos (\theta) & -\sin (\theta) \\
\sin (\theta) & \cos (\theta)
\end{array}\right]
$$

where $\alpha_{i}=0$ is the rotation angle of the quantum gate, the update process is as follows:

$\left[\begin{array}{l}\alpha^{\prime} \\ \beta^{\prime}\end{array}\right]=U(\theta)\left[\begin{array}{l}\alpha \\ \beta\end{array}\right]=\left[\begin{array}{rr}\cos (\theta) & -\sin (\theta) \\ \sin (\theta) & \cos (\theta)\end{array}\right]\left[\begin{array}{l}\alpha \\ \beta\end{array}\right]$

According to the evolutionary process, we dynamically adjust the rotation angle of quantum gate and set larger rotation angle in the algorithm of initial operation. With the increase of evolution generation, the rotation angle is gradually reduced. $\theta=k \cdot s\left(\alpha_{i}, \beta_{i}\right)$, where $k$ is a coefficient influencing the speed and $s\left(\alpha_{i}, \beta_{i}\right)$ is the direction of rotation angle. Attention: If $k$ is very large, the search step length will be very big in each iteration in the process of calculation. It is easy to fall into local extremum points. In turn, the convergence speed is slow and the computation time is too long. In this paper, $k=10 \cdot \exp (-t / T)$, where $t$ is the current evolution generation, and $T$ is the biggest evolution generation. $s\left(\alpha_{i}, \beta_{i}\right)$ is to make the algorithm to the optimal solution, the principle of which is to use the current solution to approach to the best solution best ${ }_{i}$, de-termining the direction of rotation of the quantum revolving gate. In this paper, we adopt an adjusting strategy which is general and has nothing to do with the problem [19] (Table 1).

\section{DESCRIPTION OF QAFSA}

In QAFSA, it uses qubits to encode artificial fish which mainly uses update strategy of quantum revolving gate for self-renewal, to result in a new artificial fish. Compared with BAFSA, the diversity of its fish is better. Although the size of the fish is small, it does not affect the convergence of the algorithm. And at the same time it has a faster convergence speed. High accuracy can be achieved. Patent $\mathrm{CN}$ 102,831,474 titled "Improved fuzzy C-mean clustering method based on quantum particle swarm optimization" [20]. The invention relates to a clustering method, in particu- 
Table 1. Changing the angle value.

\begin{tabular}{|c|c|c|c|c|}
\hline & \multicolumn{3}{|c|}{$s\left(\alpha_{i}, \beta_{i}\right)$} \\
\hline best $_{i}$ & $\alpha_{i} \beta_{i}>0$ & $\alpha_{i} \beta_{i}<0$ & $\alpha_{i}=0$ & 0 \\
\hline 0 & 0 & 0 & 0 & 0 \\
\hline 0 & 0 & 0 & 0 & \pm 1 \\
\hline 1 & +1 & -1 & \pm 1 & 0 \\
\hline 1 & -1 & +1 & \pm 1 & 0 \\
\hline 0 & -1 & +1 & 0 & \pm 1 \\
\hline 1 & +1 & -1 & 0 & 0 \\
\hline
\end{tabular}

lar relates to an improved fuzzy C-mean clustering method based on quantum particle swarm optimization, and belongs to the technical field of data mining and artificial intelligence. The improved fuzzy $\mathrm{C}$-mean clustering method comprises the steps of: firstly, based on the conventional fuzzy $\mathrm{C}$-mean clustering algorithm, improve the fuzzy accuracy of the conventional clustering algorithm by using a novel distance standard in place of a Euclidean standard; meanwhile classifying singly and quickly through using an AFCM (Adaptive Fuzzy C-means) algorithm to replace a randomly distributed initial clustering center to reduce the sensitivity of the clustering algorithm on the initial clustering center; and finally, introducing a QPSO (AQPSO (AdaptiveQuantum Particle Swarm Optimization)) parallel optimization concept based on distance improvement in a clustering process, so that the clustering algorithm has relatively strong overall search capability, relatively high convergence precision, and can guarantee the convergence speed and obviously improve the clustering effect.

Suppose that there is a quantum of artificial fish $Q(t)=\left\{q_{1}^{t}, q_{2}^{t} \cdots, q_{n}^{t}\right\}$, where $n$ is the population size, $t$ is evolution generation and $q_{j}^{t}$ represents a quantum artificial fish, which is used by quantum bit code: $q_{j}^{t}=\left[\begin{array}{c|c|c|c}\alpha_{1}^{t} & \alpha_{2}^{t} & \ldots & \alpha_{m}^{t} \\ \beta_{1}^{t} & \beta_{2}^{t} & \ldots & \beta_{m}^{t}\end{array}\right]$ where $m$ is the quantum number, $j=1,2, \cdots, n$. An artificial fish encoding with multiple quantum bits can be represented as [13]:

$q_{j}^{t}=\left(\begin{array}{c|c|c|c|c|c|c|c|c|c|c|c|c}\alpha_{11}^{t} & \alpha_{12}^{t} & \ldots & \alpha_{1 k}^{t} & \alpha_{21}^{t} & \alpha_{22}^{t} & \ldots & \alpha_{2 k}^{t} & \ldots & \alpha_{m 1}^{t} & \alpha_{m 2}^{t} & \ldots & \alpha_{m k}^{t} \\ \beta_{11}^{t} & \beta_{12}^{t} & \ldots & \beta_{1 k}^{t} & \beta_{21}^{t} & \beta_{22}^{t} & \ldots & \beta_{2 k}^{t} & \ldots & \beta_{m 1}^{t} & \beta_{m 2}^{t} & \ldots & \beta_{m k}^{t}\end{array}\right)$

where $m$ is variable dimension of artificial fish optimal control, $k$ is quantum bit number of optimal control variables, $\alpha_{x y}$ and $\beta_{x y}(1 \leq x \leq m, 1 \leq y \leq k)$ are complex constants and $\left|\alpha_{x y}\right|^{2}+\left|\beta_{x y}\right|^{2}=1$.

Each individual qubits coding $(\alpha, \beta)$ is initialized to $(1 / \sqrt{2}, 1 / \sqrt{2})$. A measurement is carried out for the initialization of the artificial fish in order to obtain a set of definite solution $P(t)=\left\{p_{1}^{t}, p_{2}^{t} \cdots, p_{n}^{t}\right\}$, where $p_{j}^{t}$ is $j$ solution in $t$ generation population, which is performed by binary string of length $m$, each of which is 0 or 1 and gotten by the probability of qubits. The measurement process is randomly generating a number between 0 and 1 . If this number is greater than $\alpha_{i} \beta_{i}>0$, the result value is 1 , otherwise the value is 0 . In each iteration, firstly, measure the population to get a set of determinate solutions $P(t)$, and then calculate the fitness value of the each solution. Use quantum revolving gate to adjust individual in the population to obtain the updated population, record the current optimal solution, and compare with the current target value. If it is better than the current target, let the new optimal solution as a target for the next iteration. Otherwise, keep the current target value remain the same.

\section{DESCRIPTION OF IQAFSA}

Although QAFSA can reach high precision and convergence speed, it will produce some inferior solutions while producing a better solution. The fish diversity is not very good and the convergence speed can be further improved. Therefore, the IQAFSA given in the paper is encoded by quantum bits and updated by quantum revolving gate. Make it perform preying behavior and following behavior. And swap quantum probability amplitude of artificial fish having poor results to realize the variation, then the optimal solution 
is obtained [21]. Patent CN 102,723,721 titled "Power system reactive power optimization method based on individual optimal position self-adaptive variation disturbance particle swarm algorithm" [22], the invention discloses a power system reactive power optimization method based on an individual optimal position self-adaptive variation disturbance particle swarm algorithm. The method is rapid in convergence rate, high in calculation precision and good in stability; the problem of inactive optimization of the power system can be solved effectively; and the method can be used for improving the electricity transmission efficiency of the power system and reducing the network loss configuration real time operation control in the power system.

At the early stage of the quantum artificial fish optimization, each time the artificial fish has achieved an update, it will turn into the implementation of the tailgating behavior of BAFSA, which can improve the convergence speed of artificial fish. At the late stage of the artificial fish quantum optimization, each time the artificial fish has achieved an update, it will turn into the implementation of the preying behavior of BAFSA, which can improve the accuracy of optimization. Having been updated by quantum revolving gate, preying behavior and following behavior, for those artificial fish having poor optimization results, it can increase the diversity of the artificial fish by swapping the quantum bit probability amplitude value, namely $(\alpha, \beta)$, and completely reversing the individual evolutionary direction, which can prevent the individual evolution falling into a local optimum. Finally, the variation of artificial fish is achieved. Here we use the Pauli $-X$ gate to realize mutation operation:

$$
\left[\begin{array}{ll}
0 & 1 \\
1 & 0
\end{array}\right]\left[\begin{array}{l}
\alpha \\
\beta
\end{array}\right]=\left[\begin{array}{l}
\beta \\
\alpha
\end{array}\right]
$$

Because preying and following behavior quality has close connection with vision and step of artificial fish. The results in literature [11] show that the larger the range of vision is, the stronger global search ability of artificial fish is and the faster convergence speed is. On the contrary, the smaller the range of vision is, the stronger the local search ability of artificial fish is. The larger the size of step is, the faster the convergence speed is, but there will be an oscillation phenomenon. On the other hand, the smaller the size of step is, the slower the convergence speed is, but there will be the high precision of the solution. As a result, the dynamic adjustments of artificial fish vision and step size are as follows:

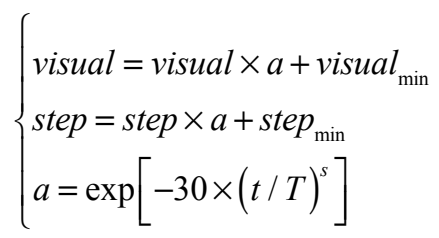

IQAFS algorithm steps are as follows:

Step 1: Initialize the quantum of artificial fish $Q\left(t_{0}\right)=\left\{q_{1}^{t_{0}}, q_{2}^{t_{0}} \cdots, q_{n}^{t_{0}}\right\}$, all optimal control variables $\left(\alpha_{i}^{t_{0}}, \beta_{i}^{t_{0}}\right)$ of the artificial fish populations are initialized to
$(1 / \sqrt{2}, 1 / \sqrt{2})$. Set up parameters such as the artificial fish vision, step length, crowded degree, maximum attempts, and evolution generation;

Step 2: Make a measurement of each of the artificial fish in the population $Q(t)$, the determinate solution $P(t)$ can be calculated;

Step 3: Conduct fitness evaluation for the determinate solutions and put the optimal artificial fish and its fitness value in the bulletin board;

Step 4:On the basis of the above adjustment strategy, firstly use the quantum revolving gate $U(\theta)$ and the corresponding rotation angle adjustment policy to update quantum bit of artificial fish, and perform preying behavior and following behavior for artificial fish through the fish evolution process, finally, new artificial fish $Q(t+1)$ is gotten;

Step 5: Perform mutation for artificial fish, choose $q(q<n)$ artificial fish having minimum fitness, and update its quantum bit probability amplitude by using formula 7 ;

Step 6: The evolution generation pluses 1 and return to step 2. When evolution generation achieves the maximum, the loop terminates and finally the optimal value is obtained.

\section{SIMULATION EXPERIMENT AND ANALYSIS}

To verify the validity of the IQAFSA, use the following four typical function simulations, and compare with BAFSA and GAFSA in literature [14].

eg.1 [14] :

$$
\begin{aligned}
\min f_{1}(x)= & \sum_{i=1}^{n}\left[x_{i}^{2}-10 \cos \left(2 \pi x_{i}\right)+10\right] \\
& \text { s.t. }-10<x_{i}<10
\end{aligned}
$$

eg.2 [14] :

$$
\begin{aligned}
\min f_{2}(x)= & \frac{1}{4000} \sum_{i=1}^{n} x_{i}^{2}-\prod_{i=1}^{n} \cos \left(\frac{x_{i}}{\sqrt{i}}\right)+1 \\
& \text { s.t. }-100<x_{i}<100
\end{aligned}
$$

eg.3 [23]:

$$
\begin{gathered}
\min f_{3}(x)=-20 \exp \left(-0.2 \sqrt{\frac{1}{n} \sum_{i=1}^{n} x_{i}^{2}}\right)-\exp \left(\frac{1}{n} \sum_{i=1}^{n} \cos \left(2 \pi x_{i}\right)\right)+20+e \\
\text { s.t. }-32<x_{i}<32
\end{gathered}
$$

eg.4 [14]:

$$
\begin{gathered}
\min f_{4}\left(x_{1}, x_{2}\right)=0.5+\frac{\sin ^{2} \sqrt{x_{1}^{2}+x_{2}^{2}}-0.5}{\left[1+0.001\left(x_{1}^{2}+x_{2}^{2}\right)\right]^{2}} \\
\text { s.t. }-100<x_{1}, x_{2}<100
\end{gathered}
$$


Table 2. Three types of index data algorithm simulation.

\begin{tabular}{|c|c|c|c|c|c|c|c|c|c|c|c|}
\hline \multirow[b]{2}{*}{ fun } & \multirow[b]{2}{*}{$\operatorname{dim}$} & \multirow[b]{2}{*}{$\max T$} & \multicolumn{3}{|c|}{ BAFSA } & \multicolumn{3}{|c|}{ GAFSA } & \multicolumn{3}{|c|}{ IQAFSA } \\
\hline & & & $A$ min & $G \min$ & $\sigma$ & $A$ min & $G \min$ & $\sigma$ & $A \min$ & $G \min$ & $\sigma$ \\
\hline \multirow{3}{*}{$f_{1}$} & 3 & 1000 & 1.492 & $1.303 \mathrm{e}-4$ & 0.62 & 1.49 & $6.526 \mathrm{e}-6$ & 2.01 & $5.413 \mathrm{e}-8$ & $5.413 \mathrm{e}-8$ & 0 \\
\hline & 5 & 1000 & 9.391 & 5.434 & 2.20 & 2.9351 & 0.995 & 1.81 & 0.3786 & $9.022 \mathrm{e}-8$ & 1.35 \\
\hline & 10 & 1500 & 50.88 & 36.7075 & 7.82 & 20.844 & 2.985 & 12.1 & 9.4 & $1.804 \mathrm{e}-7$ & 5.7 \\
\hline \multirow{3}{*}{$F_{2}$} & 3 & 1000 & 0.007 & $8.301 \mathrm{e}-4$ & $4 e-3$ & $8.5 e-3$ & $2.99 \mathrm{e}-10$ & $7 e-3$ & $8.34 \mathrm{e}-11$ & $8.3 \mathrm{e}-11$ & 0 \\
\hline & 5 & 1000 & 0.0332 & $2.24 \mathrm{e}-2$ & $4 e-3$ & $2.5 \mathrm{e}-2$ & $1.088 \mathrm{e}-8$ & 0.02 & $6.074 \mathrm{e}-3$ & $1.0 \mathrm{e}-10$ & 0.016 \\
\hline & 10 & 1500 & 0.1584 & 0.1162 & 0.03 & 3.2433 & 2.3018 & 0.73 & 0.1374 & $1.3 \mathrm{e}-14$ & 0.096 \\
\hline \multirow{3}{*}{$f_{3}$} & 3 & 1000 & 0.07 & $1.7 \mathrm{e}-4$ & 0.31 & 0.06 & $2.92 \mathrm{e}-7$ & 0.27 & $3.72 \mathrm{e}-8$ & $3.7 e-8$ & 0 \\
\hline & 5 & 1000 & 0.267 & $8.91 \mathrm{e}-4$ & 0.60 & 0.93 & $6.9 e-6$ & 0.98 & $3.72 \mathrm{e}-8$ & $3.7 \mathrm{e}-8$ & 0 \\
\hline & 10 & 1500 & 2.6388 & 2.1013 & 0.21 & 1.64 & 0.0011 & 0.93 & 0.0226 & $3.7 \mathrm{e}-8$ & 0.0278 \\
\hline$f_{4}$ & 2 & 1000 & 0.0053 & $2.4 \mathrm{e}-4$ & 0.01 & 0.0039 & $6.3 \mathrm{e}-15$ & 0.05 & $1.6 \mathrm{e}-16$ & $1.6 \mathrm{e}-16$ & 0 \\
\hline
\end{tabular}

Where fun is function, dim is dimensionmax $T$ is maximum evolution generation, $A$ min is average minimum, $G$ min is global minimum value; $\sigma$ is standard deviation respectively.

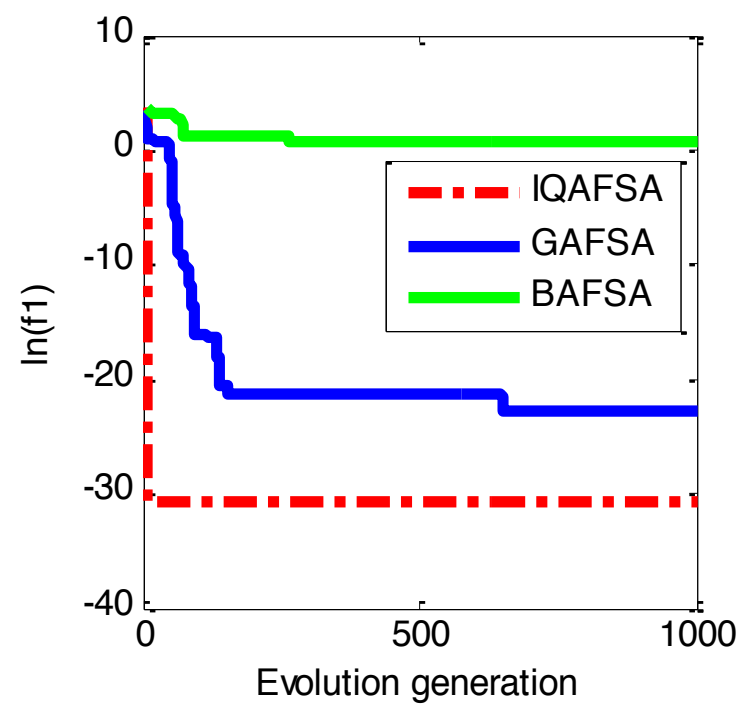

Fig. (1). Average min evolution curve 3D-function of $f_{1}$.

The theoretical minimum value of these four multidimensional functions are 0 , where $f_{4}$ is a two-dimensional function, other functions are all multi-dimensional function. Respectively, use BAFSA, GAFSA and IQAFSA to get minimum value of four different functions. Parameter settings are as follows: number of artificial fish fishnum $=40$, most test number try - number $=9$, sight radius visual $=1$, crowded degree factor $\delta=0.618$, step $=0.5$, visual ${ }_{\min }=0.001$, step $p_{\min }=$ $0.0002, s=2$. The biggest evolution generation is set to be 1000 times and 1000 times. And each algorithm runs 50 times independently. Make the global average minimum value, the global minimum value and standard deviation as a measure of the performance of algorithm, the results are as follows:

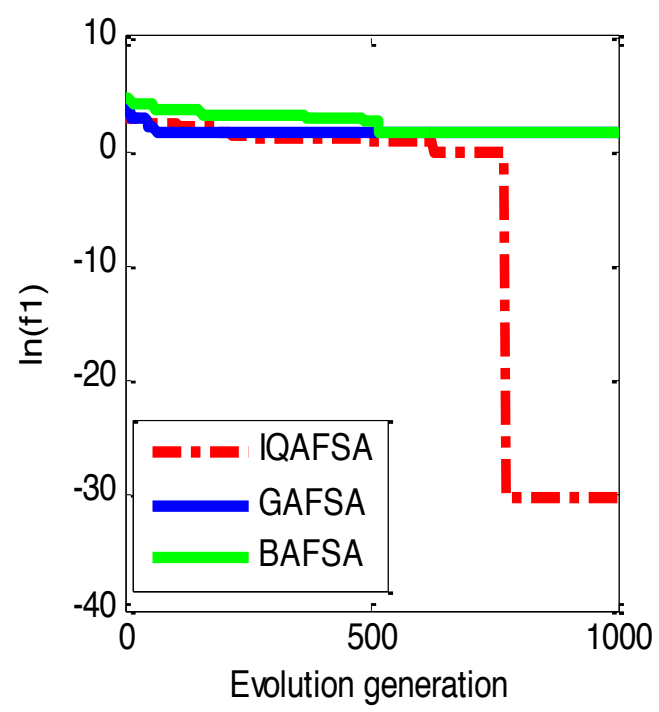

Fig. (2). Average min evolution curve 5D-function of $f_{1}$.

We use BAFSA, GAFSA, IQAFSA for 50 times and obtain the average of the evolutionary curve and draw the Figs. (1-10) of different dimension functions $f_{1}, f_{2}, f_{3}, f_{4}$. In each image, the ordinate is expressed by natural logarithm of optimal average of the function (i.e. $\ln (f)$ ), and the abscissa is expressed by evolution generation.

From Table 2, the average optimization results and the optimal results of IQAFSA are much better than that of GAFSA and BAFSA, and the precision of the optimization results is greater than GAFSA and BAFSA. In addition, most of standard deviations of IQAFSA are 0 . We can seethat stability of IQAFSA is better than that of GAFSA and BAFSA by comparing the standard deviation. 


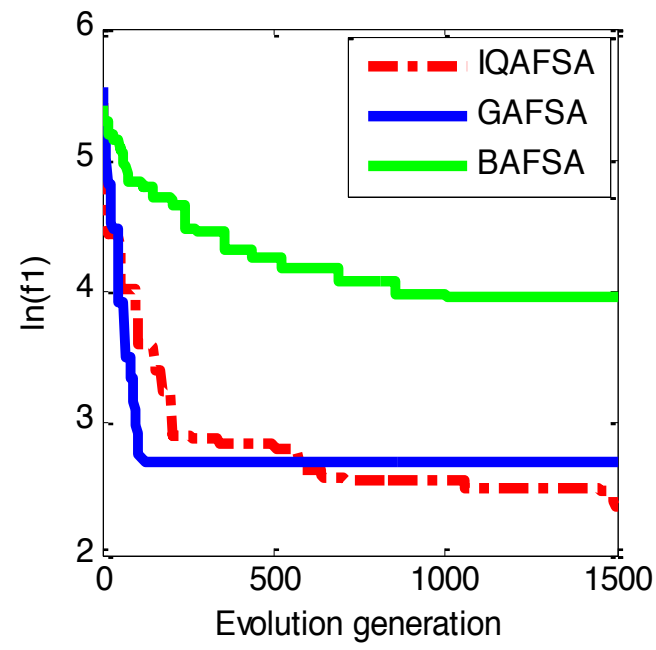

Fig. (3). Average min evolution curve 10D-function of $f_{1}$.

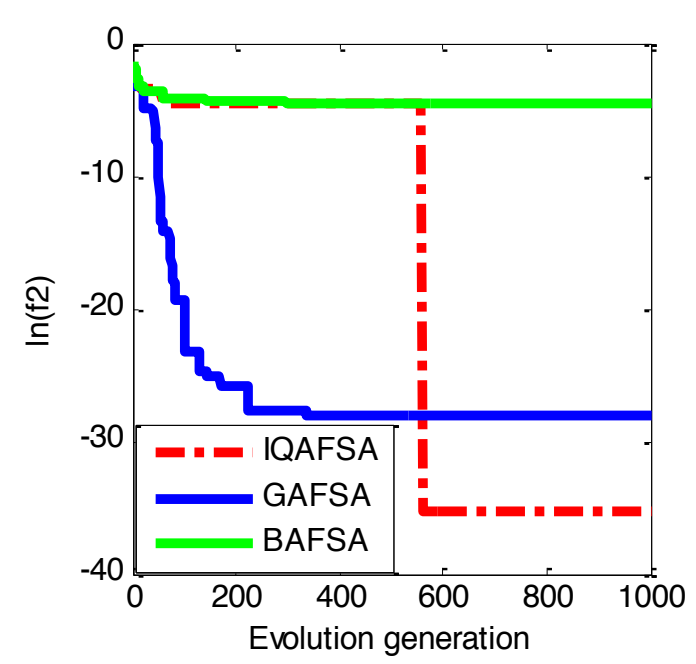

Fig. (4). Average min evolution curve 3D-function of $f_{2}$.

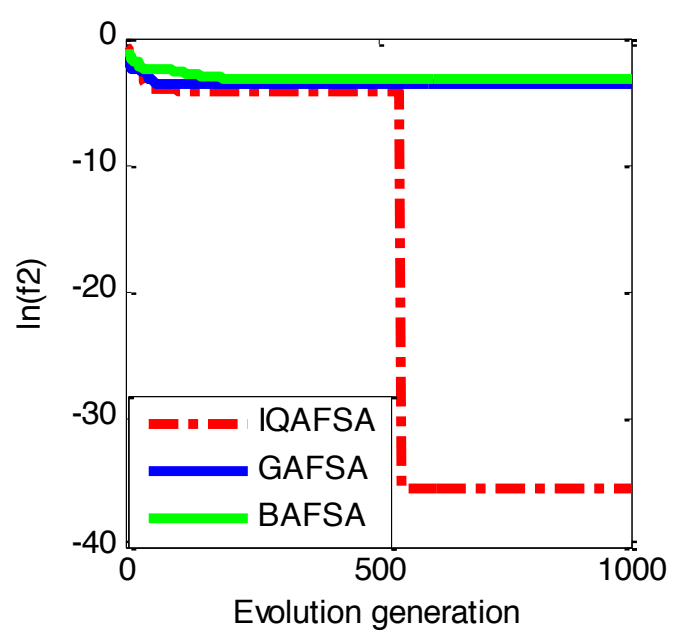

Fig. (5). Average min evolution curve 5D-function of $f_{2}$.

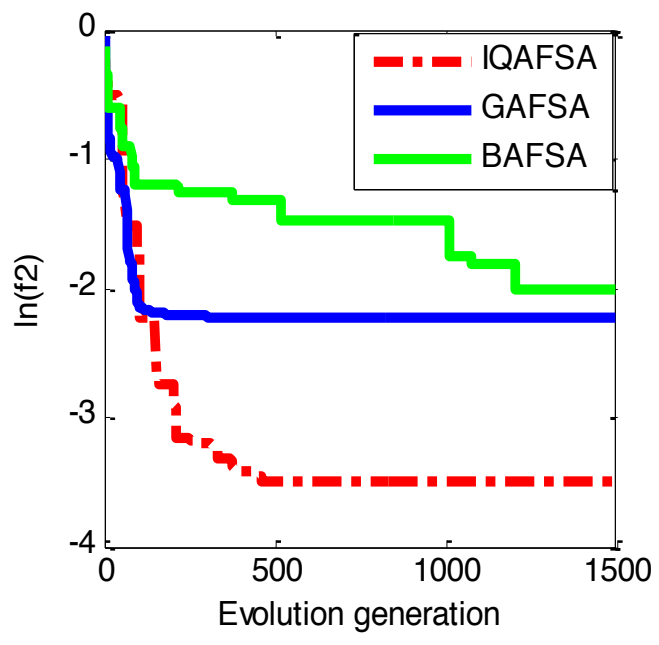

Fig. (6). Average min evolution curve 10D-function of $f_{2}$.

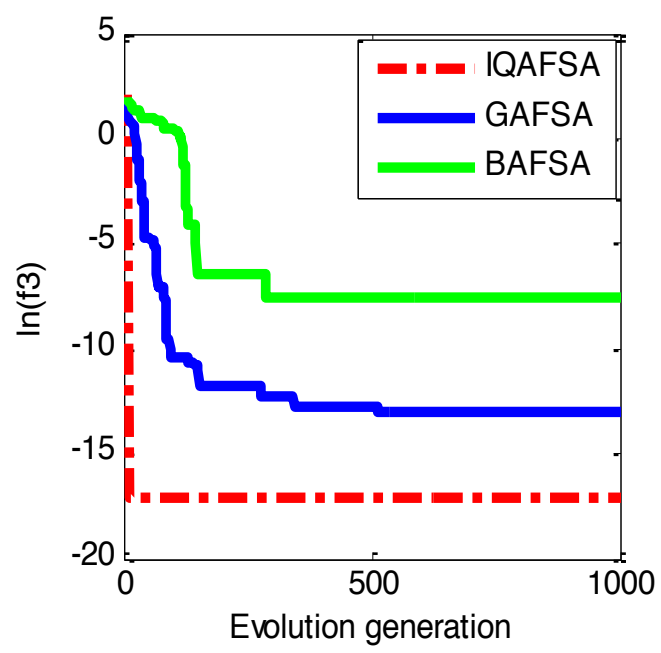

Fig. (7). Average min evolution curve 3D-function of $f_{3}$.

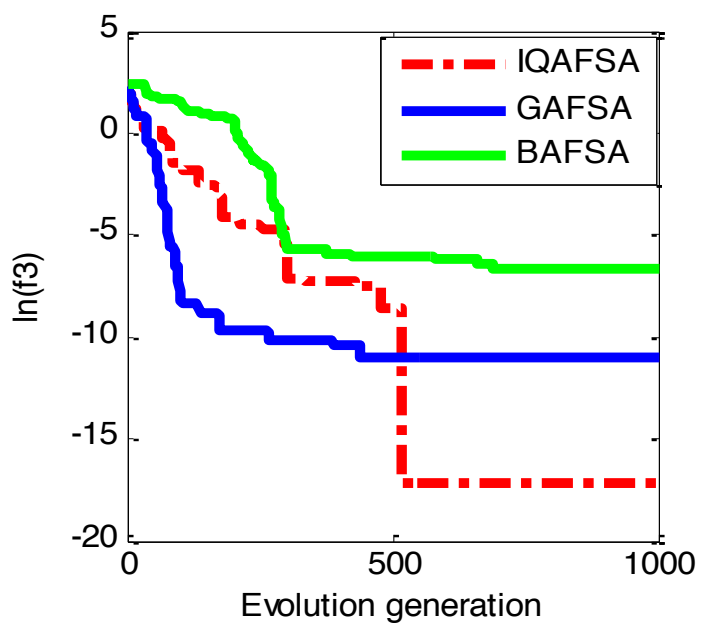

Fig. (8). Average min evolution curve 5D-function of $f_{3}$. 


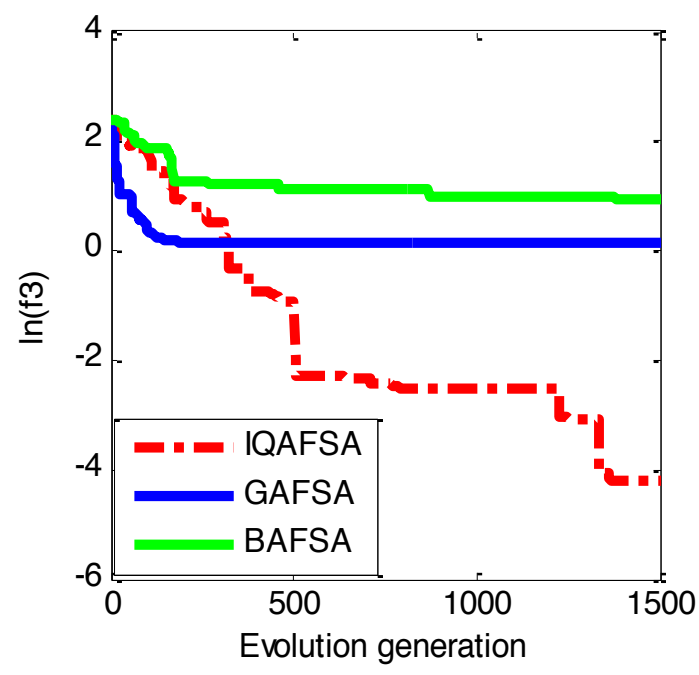

Fig. (9). Average min evolution curve 10D-function of $f_{3}$.

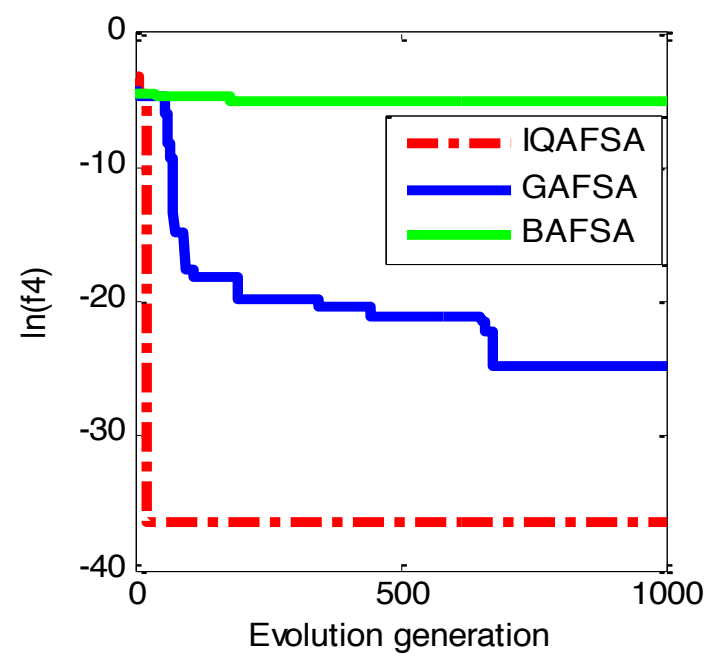

Fig. (10). Average min evolution curve 3D-function of $f_{4}$.

At the same time, from the diagram, the converge- nce speed and optimization precision of IQAFSA are obvi- ously better than that of GAFSA and BAFSA.

\section{CONCLUSION}

In this paper, quantum computing is introduced on the basis of artificial fish algorithm, and the improved quantum artificial fish algorithm is put forward. In the early stage of the artificial fish optimization, following behavior of artificial fish is added and preying behavior is added in the late stage, which improve the convergence speed and optimization precision, and enhance the diversity of population by implementation of the mutation after each iteration. Simulation results show that, compared with GAFSA and BAF-SA, IQAFSA is more suitable for solving high dimensional and complex non-convex programming problem. So the algorithm is effective and feasible. However, the theory research of IQAFSA is still in its infancy, and the convergence and stability have yet to be explored and theoretically solved, so that more detailed work and further research are needed to expand further.

\section{CONFLICT OF INTEREST}

The authors confirm that this article content has no conflict of interest.

\section{ACKNOWLEDGEMENTS}

This work was supported by Hubei Province Key Laboratory of Systems Science in Metallurgical Process of China under Grant Z201402, the Natural Science Foundation $\mathrm{f} \mathrm{Hu}$ bei Province, China under Grant 2013CFA131, and the National Natural Science foundation of China under Grant 61374028 .

\section{REFERENCES}

[1] T. S. Du, P.S. Fei, and Y. J. Shen."A modified niche genetic algorithm based on evolution gradient and its simulation analysis," IEEE International Conference on Natural Computation, IEEE Press, Haikou,. vol.4, pp. 35-39, 2007.

[2] H. C. Tsai, and Y. H. Lin."Modification of the fish swarm algorithm with particle swarm optimization formulation and communication behavior," Applied Soft Computing, vol.11, no. 8, 2011, pp. 5367-5374.

[3] A. M. A .C. Rocha, T. F. M .C. Martins, and E. M. G. P. Fernandes."An augmented lagrangian fish swarm based method for Global Optimization," Journal of Computational and Applied Mathematics, vol.235, no. 16, 2011, pp. 4611-4620.

[4] Y. F. Hu, X. X. Li, X. H. Zhang, Y. H. Zhang, X. Wang, X. Yu, Y. Guo, J. H. Su. "Dispatching function optimization method based on multi-objective genetic algorithm," C.N. Patent 102,708,248 Oct 3, 2012.

[5] X. L. Li, Z .J. Shao, and J. X. Qian.”An optimization model based on animal commune: fish swarm algorithm, "Systems Engineering and Practice, vol. 22, no. 11, pp. 32-38, 2002.

[6] L. Gyongyosi , and S .Imre."Algorithmic superactivation of asymptotic quantum capacity of zero-capacity quantum channels," Information Sciences, vol. 222, no .10, pp.737-753, 2013.

[7] Hotaling, P. Steven," General purpose quantum computing, "U.S. Patent 5,940,193, Aug 17,1999.

[8] J. Sun, W. Chen, W. Fang, X. J. Wun, and W. B. Xu. "Gene expression data analysis with the clustering method based on an improved quantum-behaved particle swarm optimization," Engineering Applications of Artificial Intelligence, vol. 25, no. 2, pp. 376391, 2012

[9] J. X. V. Neto, D. L. An. Bernert, and L. S. Coelho.”Improved quantum-inspired evolutionary algorithm with diversity Information applied to economic dispatch problem with prohibited operating Zones,"Energy Conversion and Management, vol.52, no. 1, pp.8-14, 2011.

[10] Goto, H. Ichimura, and Kouichi, "Quantum computing method and quantum computer", U.S. Patent 7,447,719, Nov 4,2008.

[11] E. Rjeffel, and W. Polak."An introduction to quantum computing for non-physicists," ACM Computing Surveys, vol.32, n.o 3, pp.300-335, 2000.

[12] B. Qiao, and H. E. Ruda "Nonlinear liouville equation, project green function and stabilizing quantum computing," Statistical Mechanics and its Applications, vol. 333, no. 15, pp. 197-224, 2004

[13] Koza, and R. John, "Non-linear genetic algorithms for solving problems," U.S. Patent 4,935,877, Jun 19,1990.

[14] L. G. Wang, Y. Hong, and Q. H. Shi. "The global edition artificial fish swarm algorithm," Journal of System Simulation, vol.21, no. 23, pp. 7483-7502, 2009.

[15] M. Y. Jiang, Y. M. Cheng, and D. F. Yuan. "Artificial fish-swarm algorithm based on overall information," C.N. Patent 101,515,338 Oct 26, 2009 
[16] Ulyanov, S. V. Panfilov, and A. Sergey, "Efficient simulation system of quantum algorithm gates on classical computer based on fast algorithm," U.S. Patent 20,060,224,547, Oct 5, 2006.

[17] P. C. Li, and S .Y. Li. "Learning algorithm and application of quantum BP neural networks based on universal quantum gates," Journal of Systems Engineering and Electronics, vol.19, no. 1, pp.167$174,2008$.

[18] D. Bhowmik, S. Bandyopadhyay. "Gate control of the spinsplitting energy in a quantum dot: application in single qubit rotation," Low-dimensional Systems and Nanostructures, vol.41, no 4, pp. 587-592, 2009.

[19] T. Radtke, S. Fritzsche. "Simulation of n-qubit Quantum Systems. I. Quantum Registers and Quantum Gates," Computer Physics Communications, vol.173, no. 1, pp. 91-113, 2005.
[20] L. Mao, and Y. Lin, "Improved fuzzy C-mean clustering method based on quantum particle swarm optimization," C.N. Patent 102,831,474, Dec 19, 2012.

[21] M. J. Qi, P.Y. Wang, " Quantum artificial fish algorithm," Anhui Agricultural Sciences, vol.40, no. 8, pp. 4469-4472, 2012.

[22] Z. G. Liu, J. J. Zeng, J. Liu, "Power system reactive power optimization method based on individual optimal position self-adaptive variation disturbance particle swarm algorithm," C.N. Patent 102,723,721 Oct 10, 2012.

[23] Y. Tan, G.Z. Tan, "A chaotic local search strategy of differential evolution algorithm for global optimization," Computer engineering and its application, vol. 45, no. 14, pp. 1002-8331, 2009.

Received: July 23, 2014

(C) Hu et al.; Licensee Bentham Open.

This is an open access article licensed under the terms of the Creative Commons Attribution Non-Commercial License (http://creativecommons.org/licenses/by-nc/3.0/) which permits unrestricted, non-commercial use, distribution and reproduction in any medium, provided the work is properly cited. 